\title{
3D 打印技术在有机合成化学中的应用
}

\author{
赖石林 ${ }^{a}$ 廖 旭 ${ }^{a}$ 张 辉 ${ }^{a}$ 蒋 岩 ${ }^{a}$ \\ 刘源岗*,b王士斌 $a$ 熊兴泉*,a \\ ( ${ }^{a}$ 华侨大学材料科学与工程学院 厦门 361021) \\ ( ${ }^{b}$ 华侨大学化工学院 厦门 361021)
}

\begin{abstract}
摘要 相对于传统的材料去除一切削加工技术, 3D 打印作为一种 “ 自下而上” 的材料累加制造方法, 不仅操作简单、 制造成本更低以及可实现快速生成，而且可实现复杂结构件的精确定制. 因此，3D 打印已成为第三次工业革命的代表 性技术. 近年来, 化学家们将 3D 打印技术与有机合成相结合, 在多通道异相催化剂和新型反应装置的研发设计等方面 取得了积极的进展，进而使这项技术在有机合成领域中应用越来越广泛. 对近年来基于 $3 \mathrm{D}$ 打印技术的有机合成研究进 展进行了综述, 涵盖基于 3D 打印异相催化剂或催化剂载体、3D 打印反应装置和 3D 打印流反应器, 并对该领域的发 展趋势进行了展望.
\end{abstract}

关键词 3D 打印技术; 3D 打印催化剂; 3D 打印反应器; 有机合成

\section{Application of 3D Printing Technology in Organic Synthetic Chemistry}

\author{
Lai, Shilin ${ }^{a}$ \\ Liao, $\mathrm{Xu}^{a}$ \\ Zhang, Hui ${ }^{a}$ \\ Jiang, $\operatorname{Yan}^{a}$ \\ Liu, Yuangang ${ }^{*, b}$ Wang, Shibin ${ }^{a} \quad$ Xiong, Xingquan ${ }^{*, a}$ \\ ( ${ }^{a}$ College of Materials Science and Engineering, Huaqiao University, Xiamen 361021) \\ ( ${ }^{b}$ College of Chemical Engineering, Huaqiao University, Xiamen 361021)
}

\begin{abstract}
Compared with traditional material removal-cutting method, 3D printing is a "bottom-up" material accumulation manufacturing technology. This novel technology is not only simple to operate, but also has a lower manufacturing cost and can be quickly generated. What's more, 3D printing technology can fabricate the be-spoke objects with intricate internal structures. Therefore, 3D printing has been a representative technology of the third industrial revolution. In recent years, chemists have combined 3D printing technology with organic synthesis and made many good achievements in the development of new multichannel heterogeneous catalysts and reaction devices, which has made this technology more and more widely used in the field of organic synthesis. In this review, the progress of the organic synthesis based on 3D printing technology from 2012 to 2018 are summarized, such as 3D-printed heterogeneous catalysts, 3D-printed devices and 3D-printed continuous flow microreactors. Furthermore, the development trends of this field in the future are also prospected.
\end{abstract}

Keywords 3D-printed technology; 3D-printed heterogeneous catalysts; 3D-printed microreactors; organic synthesis

自 Hull ${ }^{[1]}$ 于 1986 年发明了第一台立体平版印刷 3D 打印机以来, 3D 打印技术经过数十年的研究与发展得 到了长足的进步 ${ }^{[2]}$, 并已广泛应用于制造工业 ${ }^{[3,4]}$ 、教

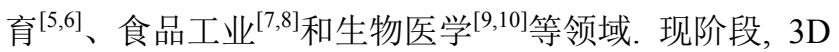
打印技术的发展主要体现在 3D 打印材料和 3D 打印工
艺两方面, 具体情况如 Figure 1 所示. 3D 打印技术的原 理是先通过计算机辅助设计(CAD)或计算机动画建模软 件建模，再将建成的三维模型 “分区”成逐层的截面，并 以光固化立体造型术(STL)的标准文件格式保存, 然后 3D 打印机通过读取文件中的横截面信息, 用液体状、粉

\footnotetext{
* Corresponding authors. E-mail: ygliu@hqu.edu.cn; xxqluli@hqu.edu.cn

Received January 2, 2019; revised March 12, 2019; published online March 29, 2019

Project supported by the Natural Science Foundation of Fujian Province (No. 2016J01063), the Program for New Century Excellent Talents in Fujian Province (No. 2012FJ-NCET-ZR03) and the University Distinguished Young Research Talent Training Program of Fujian Province (No. 11FJPY02), the National Marine Economic Innovation and Development Project (No. 16PYY007SF17) and the Subsidized Project for Postgraduates' Innovative Fund in Scientific Research of Huaqiao University.

福建省自然科学基金(No. 2016J01063)、福建省 “高校新世纪优秀人才支持计划” (No. 2012FJ-NCET-ZR03)和福建省 “高校杰出青年科研人才培育计 划” (No. 11FJPY02)、“十三五” 海洋经济创新发展示范(No. 16PYY007SF17)以及 “华侨大学研究生科研创新基金” 资助项目.
} 
状或片状的材料将这些截面逐层打印、制造出一个三维 实体 ${ }^{[1]}$. 该技术最大的优势在于能够直接将三维数据模 型进行实体建造, 从而为异相催化剂、微反应器的一步 快速制造工艺提供了可能性. 目前, 在一步快速制造异 相催化材料、微反应器方面, 3D 打印技术已成为新的研 究热点. 与传统材料加工技术相比, $3 \mathrm{D}$ 打印技术有诸多 突出优势: (1)可实现数字化制造; (2)可使三维结构的物 体先分解成二维层状结构, 逐层累加形成三维物品, 从 根本上解决传统制造受制于模具的缺陷; (3)可利用 “从 下而上” 的堆积方式, 省去了通道密封的粘结环节; (4) 有利于个性化订制, 环保且低能耗 ${ }^{[12,13]}$. 因此, 利用 3D 打印技术制备异相催化材料、微反应器方面具有巨大的 前景, 可大大缩短研发的时间, 快速实现从构想的三维 数字模型到实体原型, 并根据原型测试对初始设计进行 及时的信息反馈, 以最终得到优化的异相催化材料、微 反应器设计, 这些优势对于新的合成方法与合成技术的 更新尤为重要. 最近几年, 化学合成领域对这一革命性 技术的应用日益紧密, 怎样快速、高效地将 3D 打印技 术和化学反应的催化剂、微反应器的制备相结合以及开 发更多的 3D 打印材料应用与合成化学领域, 是现代合 成化学家遇到的前所未有的机遇和挑战 ${ }^{[14]}$. 本文综述 了有机合成化学中 $3 \mathrm{D}$ 打印技术的应用进展, 特别是基 于 3D 打印的异相催化材料、反应装置和微流反应器三 方面.

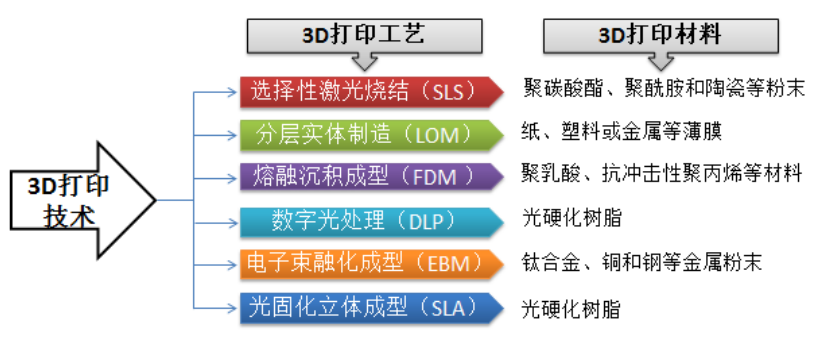

图 $13 \mathrm{D}$ 打印技术

Figure $13 \mathrm{D}$ printing technology

\section{3D 打印异相催化剂或异相催化剂载体}

2016 年, Sotelo 等 ${ }^{[15]}$ 利用 3D 打印技术首次合成了 圆柱多孔结构的 $\mathrm{Cu} / \mathrm{Al}_{2} \mathrm{O}_{3}$ 催化剂. 该催化剂具有高机械 强度和比表面积, 而且其孔隙率可控. 首先, 将一定量 $\mathrm{Al}_{2} \mathrm{O}_{3}$ 粉末加入到 $\mathrm{Cu}\left(\mathrm{NO}_{3}\right)_{2}$ 溶液中, 随后加入粘度调节 剂羟丙基甲基纤维素形成悬浮液, 再将聚梄酰亚胺 (PEI, $M_{\mathrm{w}}=2000$ )与其混合, 经离心、浓缩得到 $\mathrm{Cu} / \mathrm{Al}_{2} \mathrm{O}_{3}$ 水性油墨, 于 3D 打印机中打印出相应的圆柱形状多孔 结构材料, 并经 $1400{ }^{\circ} \mathrm{C}$ 烧结后可制备出 $\mathrm{Cu} / \mathrm{Al}_{2} \mathrm{O}_{3}$ 催化 剂. 在碱性以及 $80{ }^{\circ} \mathrm{C}$ 条件下, 以 $\mathrm{N}, \mathrm{N}$-二甲基甲酰胺 (DMF)为溶剂, 采用 $3 \mathrm{D}$ 打印制备的 $\mathrm{Cu} / \mathrm{Al}_{2} \mathrm{O}_{3}$ 为催化剂,
将卤代苯分别与咪唑、苯并咪唑、苯甲酰胺或对澳苯甲 酰胺等反应，高效率合成出一系列 $N$-芳基咪唑或 $N$-芳 基苯并咪唑类化合物。研究表明, 该种催化剂对 Ullmann 反应具有非常高的催化活性(Figure 2), 而且避 免了因金属 $\mathrm{Cu}$ 的浸出而造成产物的污染.

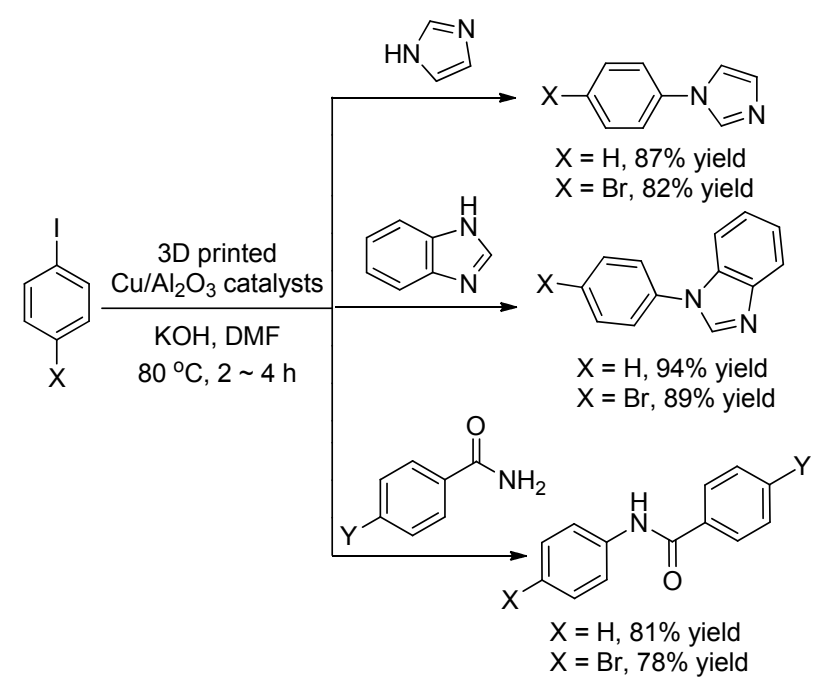

图 $23 \mathrm{D}$ 打印 $\mathrm{Cu} / \mathrm{Al}_{2} \mathrm{O}_{3}$ 催化剂及其催化的 Ullmann 反应 Figure $23 \mathrm{D}$ printed $\mathrm{Cu} / \mathrm{Al}_{2} \mathrm{O}_{3}$-catalyzed Ullmann reactions

随后, Sotelo 团队 ${ }^{[16]}$ 又报道了采用 $3 \mathrm{D}$ 打印技术制备 了另外一种具有多孔性圆柱形网状结构的 $\mathrm{Al}_{2} \mathrm{O}_{3}$ 异相催 化剂. 该催化剂以 $\mathrm{Al}_{2} \mathrm{O}_{3}$ 粉末为原料, 再将其与水、分散 剂 Darvan 821A、羟丙基甲基纤维素以及 PEI $\left(M_{\mathrm{w}}=\right.$ 2000)混合, 形成的 $\mathrm{Al}_{2} \mathrm{O}_{3}$ 胶体油墨通过 3D 打印技术打 印出构型后, 经过高温 $\left(1500{ }^{\circ} \mathrm{C}\right)$ 烧结而成. $\mathrm{Al}_{2} \mathrm{O}_{3}$ 异相 催化剂可作为一种 Lewis 酸催化多组分反应, 尤其对在 无溶剂和微波辐射条件下进行的 Biginelli 反应和 Hantzsch 反应具有非常好的催化效果，同时具有可回收 性好、反应时间短和高产率等特点. 研究发现, 当温度 为 $100{ }^{\circ} \mathrm{C}$ 时, 该催化剂可促进醛、二羰基化合物和(硫) 腿衍生物三组分间的 Biginelli 反应, 进而合成具有生物 活性的 3,4-二氢嘧啶-2(1H)-酮类化合物 $(9 \mathrm{~min}, 84 \%$ $96 \%$ ); 而当温度升到 $120{ }^{\circ} \mathrm{C}$ 时，该催化剂可促进醛、 $\alpha$ 二羰基化合物和乙酸铵三组分间的 Hantzsch 反应, 生成 具有生物活性的 $1,4-$ 二氢吡啶衍生物 $(0.5 \sim 1 \mathrm{~h}, 78 \%$ 93\%) (Figure 3).

2017 年, Manzano 团队 ${ }^{[17]}$ 报道了一种能够高效制 备具有化学催化活性结构的 3D 打印新方法. 这一新打 印方法是以光聚合多功能分子为打印材料, 经过光固化 SLA工艺打印而成. 该团队用丙烯酸分别与聚乙二醇二 丙烯酸酯(PEDGA)、PEDGA 和烯丙胺(或己二烯胺)、 PEDGA 和丙烯酸铜以及 BAPO 混合, 依次形成 3 种不 同的 3D 打印液体油墨后, 打印出了 $\mathrm{AL}-(\mathrm{COOH})_{2} \mathrm{Cu}$ 、 


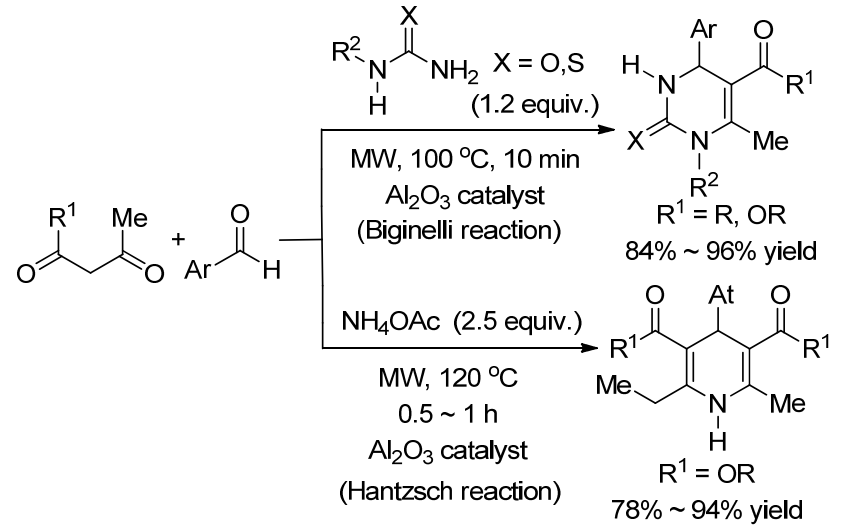

图 $33 \mathrm{D}$ 打印 $\mathrm{Al}_{2} \mathrm{O}_{3}$ 催化的 Bigineeelli 和 Hantzsch 反应

Figure 3 Bigineeelli and Hantzsch reactions catalyzed by $3 \mathrm{D}$ printed $\mathrm{Al}_{2} \mathrm{O}_{3}$

AL-COOH 和 AL-COOH-co- $\mathrm{NH}_{2}$ 催化剂. 此外, 该团队 将这 3 种催化剂依次进行了苯乙炔和(叠氮甲基)苯二组 分间的 Huisgen 环加成反应, 苯甲醛和苯胺、环己酮三 组分间的 Mannich 反应，4-硝基苯甲醛和丙酮二组分间 的羟醛缩合反应(Figure 4). 结果表明, 该种能够制备出

(a)<smiles>C#Cc1ccccc1</smiles><smiles>N#CC(=O)OCC(=O)O</smiles><smiles>c1ccc(Cn2cc(-c3ccccc3)nn2)cc1</smiles>
$3.83 \sim 5.77 \mathrm{~h}^{-1}$ TOF

(b)<smiles>O=Cc1ccccc1</smiles><smiles>Nc1ccccc1</smiles><smiles>O=C1CCCCC1C(C(=O)O)C(=O)O</smiles><smiles>c1ccccc1</smiles><smiles>C=CCNC(c1ccccc1)C1CCCCC1=O</smiles>

$65 \% \sim 75 \%$ yield

(c)

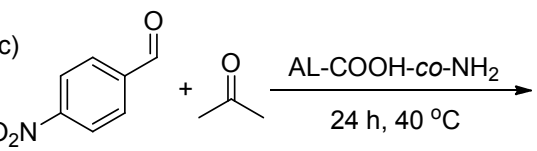<smiles>CC(=O)CC(O)c1ccc([N+](=O)[O-])cc1</smiles><smiles>CC(=O)/C=C/c1ccc([N+](=O)[O-])cc1</smiles>

图 43 种不同催化剂 $\mathrm{AL}-(\mathrm{COOH})_{2} \mathrm{Cu}, \mathrm{AL}-\mathrm{COOH}, \mathrm{AL}-\mathrm{COOH}-$ $c o-\mathrm{NH}_{2}$ 催化的(a) Huisgen 环加成反应、(b) Mannich 反应、(c) 着醛缩合反应

Figure 4 Huisgen cycloaddition (a), Mannich (b) and aldol-condensation (c) reactions catalyzed by AL- $(\mathrm{COOH})_{2} \mathrm{Cu}, \mathrm{AL}-$ $\mathrm{COOH}, \mathrm{AL}-\mathrm{COOH}-\mathrm{co}-\mathrm{NH}_{2}$ respectively
催化活性结构的 3D 打印新方法非常适合制造一些应用 于化学合成、传感和分离等前沿任务的复杂功能装置.

2018 年, Díaz-Marta 团队 ${ }^{[18]}$ 报道了一种基于 3D 打 印技术制备出可用于多组分催化的层状多孔圆柱形 3D$\mathrm{Pd} / \mathrm{Cu}$ 多相催化剂. 首先, 将一定量 $\mathrm{SiO}_{2}$ 粉末、PVBPVAPVAc 和 PEG $\left(M_{\mathrm{w}}=600\right)$ 与 2-丙醇溶液混合制备 $\mathrm{SiO}_{2}$ 胶体油墨, 然后经 3D 打印成型后, 干燥并脱脂, $1500{ }^{\circ} \mathrm{C}$ 下烧结 $3 \mathrm{~h}$ 形成异相催化剂载体. 将该载体表面 活化后，依次与(3-氨基丙基)三甲氧基硅烷(APTS)或 [3-(2-氨基乙基氨基)-丙基]三甲氧基硅烷(AAPTS)进行 氨基功能化修饰, 再将氨基改性后的多通道 $\mathrm{SiO}_{2}$ 载体 与 $\mathrm{CuI}$ 或 $\mathrm{Pd}(\mathrm{AcO})_{2}$ 进行络合, 最后得到 $3 \mathrm{D}-\mathrm{SiO}_{2}-\mathrm{APTS}-$ $\mathrm{Cu} 、 3 \mathrm{D}-\mathrm{SiO}_{2}-\mathrm{AAPTS}-\mathrm{Pd}$ 两种目标异相催化剂. 该团队 将这两种催化剂组合在一起, 研究了以 $t-\mathrm{BuOH} / \mathrm{H}_{2} \mathrm{O}$ 为 溶剂、基于铜催化的炔-叠氮化物环加成 $(\mathrm{CuAAC})$ 和钯 催化交叉偶联(PCCC) 反应的非均相多组分催化反应 (MMCR), 实现了多种 1,2,3-三唑衍生物(78\% 88\%)的 快速合成(Figure 5). 结果表明, 该催化剂具有非常好的 稳定性和高的催化活性，可循环使用多次且能保持高的 产率.

\section{3D 打印反应装置}

2012 年, Cronic 团队 ${ }^{[19,20]}$ 首次报道出一种可重复使 用的 3D 打印反应器. 这一反应器是用乙酰氧基硅酮密 封胶作为 $3 \mathrm{D}$ 打印材料打印而成的, 且它是由两个溶液 储存室、一个反应室和一个铟锡氧化物构成的观察窗口 组成. Cronic 团队用此反应器进行了以 4-甲氧基苯胺与 5-(2-溴乙基)菲啶-5-溴化物为原料的菲啶基杂环化合物 的合成研究(Figure 6). 结果表明，该合成方法不仅可获 得高产率的目标产物, 而且反应器体积的变化会使目标 产物发生改变 $\left(V_{\text {reactor }}=9.0 \mathrm{~mL}, \mathrm{C}_{22} \mathrm{H}_{19} \mathrm{BrN}_{2} \mathrm{O},>90 \%\right.$; $V_{\text {reactor }}=2.0 \mathrm{~mL}, \mathrm{C}_{22} \mathrm{H}_{20} \mathrm{~N}_{2} \mathrm{O}, 80 \%$ ). 之后, 他们在用甲苯 稀释的乙酰氧基硅酮密封胶中加入 $\mathrm{Pd} / \mathrm{C}$ 形成合适的打 印油墨，然后利用相同的方法打印了一种具有催化作用 的相似反应装置. 当溶剂为甲醇、反应时间为 $30 \mathrm{~min}$ 和 $\mathrm{Et}_{3} \mathrm{SiH}$ 作为氢源的条件下, 此装置实现了苯乙烯到乙苯 定量转化.

2013 年, Cronic 团队 ${ }^{[21 ~ 23]}$ 报道了一种集试剂、催化 剂和纯化装置于一体的密闭反应装置. 该装置为 “ $L$ ” 型，由一个色谱柱和 4 个长为 $20 \mathrm{~mm}$ 的立方体通过圆形 管道连接组成，且这 4 个立方体分别是 1 个原始物料室 和 3 个反应室. 这 3 个反应室进行的反应依次分别是: (1)丙烯醛和取代环戊二烯的 Diels-Alder 环化反应; (2) Diels-Alder 环化产物和苯胺生成亚胺的反应; (3)亚胺和 氢气在 $\mathrm{Pd} / \mathrm{C}$ 进行的还原反应(Figure 7). 操作人员只需 


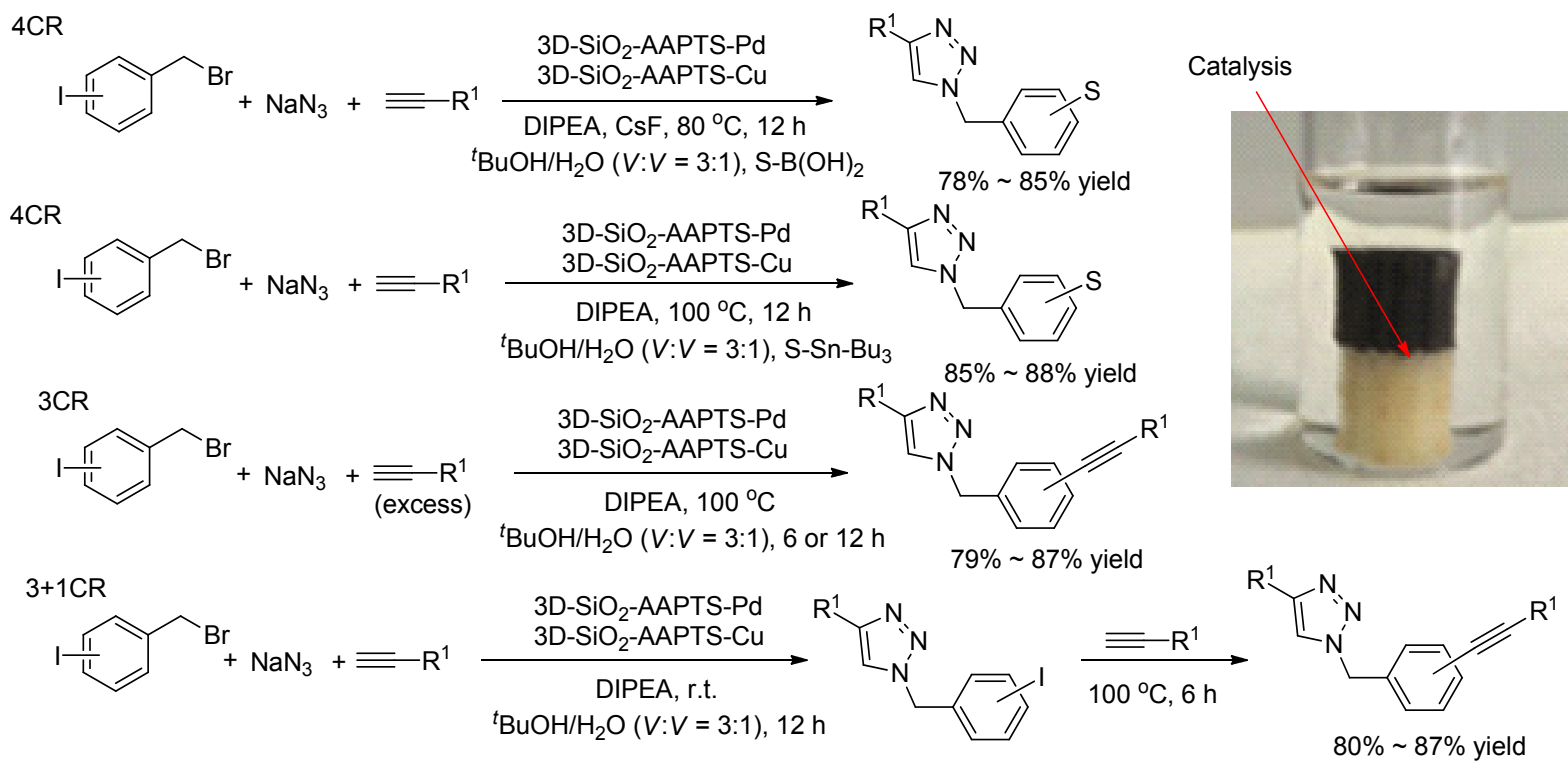

图 $5 \mathrm{Pd}-\mathrm{Cu}$ 催化剂的多组分催化反应概述( $\mathrm{Cu}-\mathrm{Pd} \mathrm{MMCRs})$

Figure 5 General outline for the strategy of $\mathrm{Cu}-\mathrm{Pd}$ MMCRs
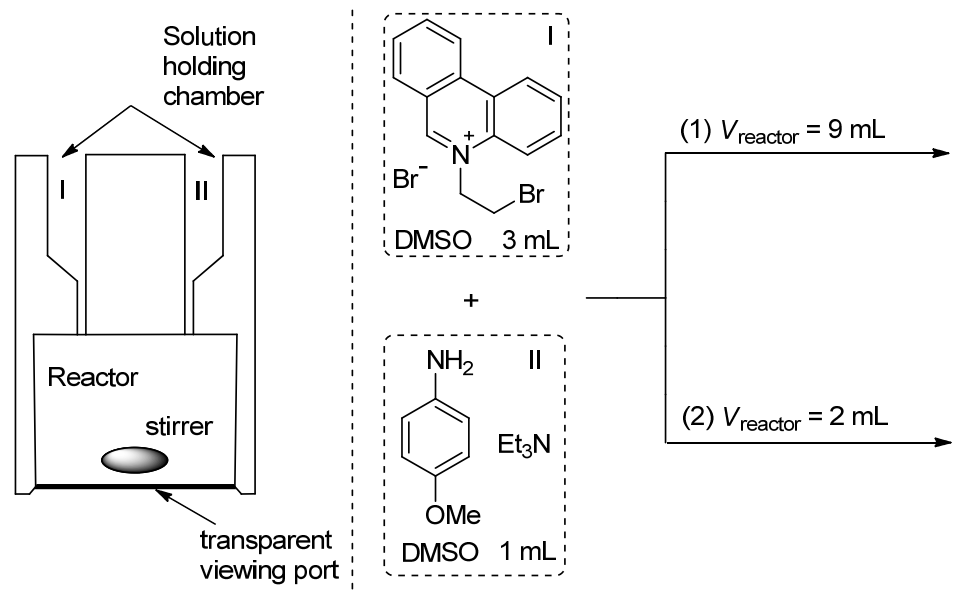

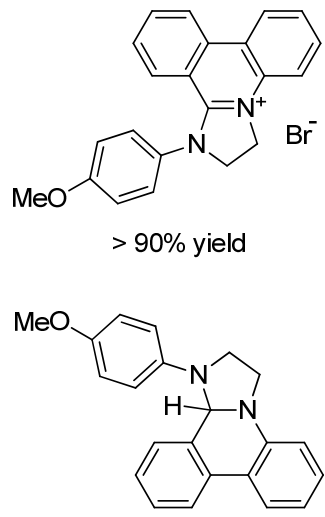

$80 \%$ yield

图 $63 \mathrm{D}$ 打印反应器中菲啶基杂环化合物的选择性合成

Figure 6 Selective synthesis of phen-anthridine-based heterocycles in 3D printing reactor

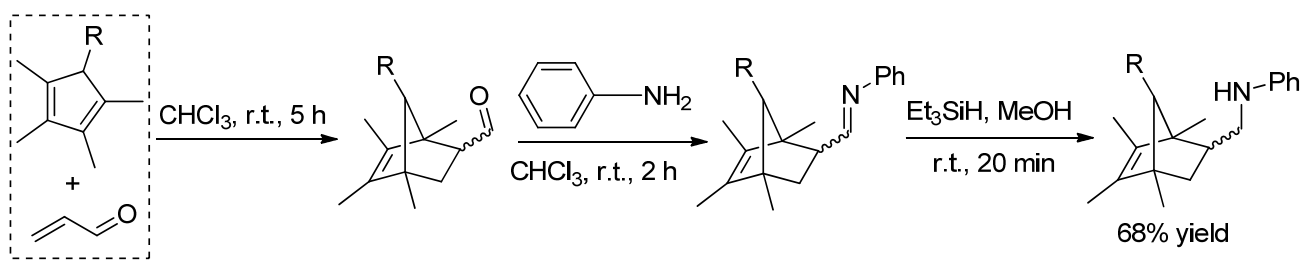

图 $73 \mathrm{D}$ 打印顺序反应器中进行的多步反应过程示意图

Figure 7 The multi-step reaction process performed in the 3D-printed sequence reactor

要通过简单的旋转操作就可在不需要葲的情况下完成 此多步反应, 且该多步反应最终产品的产率可以达到 $68 \%$.

2016 年, Cronic 团队 ${ }^{[24]}$ 设计了一台用于合成外消旋 布洛芬的自动化 3D 打印装置. 该设备中进行的所有反
应过程都是在常温常压下，而且整个反应过程都可通过 计算机软件来控制. 首先, 该设备根据设计好的反应器 图纸打印出反应器. 然后, 计算机软件按照 Figure 8 所 示的布洛芬合成路线来控制每种化学试剂的添加以完 成整个合成过程. 结果表明, 在无操作人员介入下, 最 
终产品的产率可达到 34\%.

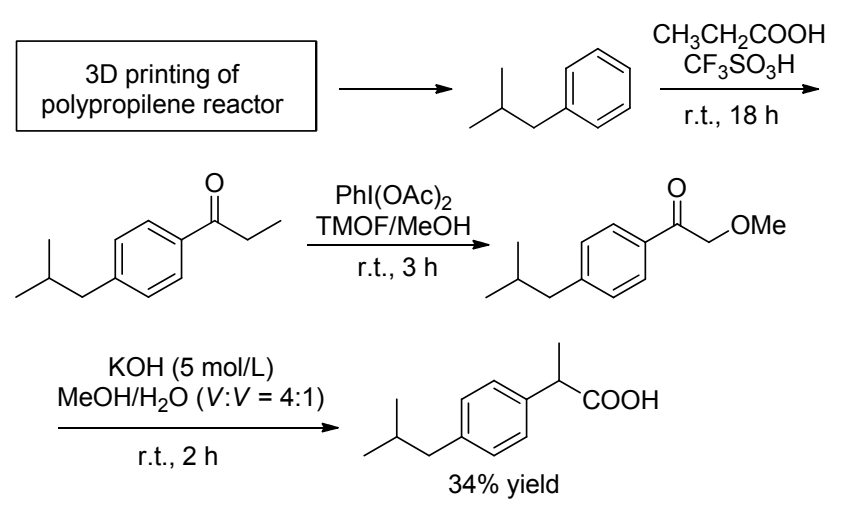

图 83 D 打印聚丙烯反应器中外消旋布洛芬的合成

Figure 8 Synthesis of racemic ibuprofen in 3D-printed polypropilene reactor

2016 年, Ananikov 等 ${ }^{[25]}$ 报道了一种可促进可见光介 导的无金属颈基-炔点击反应的光致反应器. 该反应器 包含一个 LED 光源, 主要进行的是炔烃和硫醇在曙红 Y 催化下的一个光还原反应. 值得注意的是, 该情况下的 反应不仅具有非常高的原子经济效益和高达 $91 \%$ 的产 品收率, 而且对该产物的 $E$-异构体具有极高的选择性 (高达 $98 ：$ 2) (Figure 9).

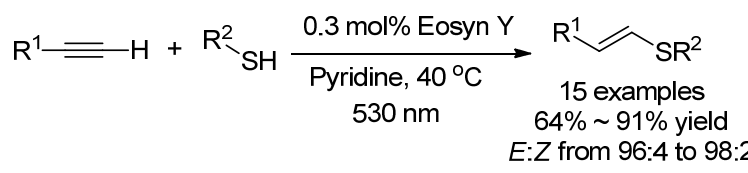

图 $93 \mathrm{D}$ 打印光反应器以及光致无金属硫醇一炔点击反应 Figure 9 Image of 3D printed photo-reactor and photoinduced metal-free thiol-acetylene click reaction

2017 年, Ananikov 等 ${ }^{[26,27]}$ 利用不同材料打印出了一 系列的标准反应器, 并且在此基础上研究了它们的抗压 能力、在不同溶剂中的稳定性以及抵抗真空泄露的能力. 研究发现, 运用于有机合成过程中的 $3 \mathrm{D}$ 打印反应器的 材料的适用性具有如下规律: PP $>$ PLA $>$ ABS $>$ PETG. 除此之外, Ananikov 等使用这些 3D 打印反应器进行了 1-溴-4-甲基苯与苯硣酸两组分间的 Suzuki-Miyaura 交叉 偶联反应(产率在 42 79\%之间)和在 $\mathrm{Ni}$ (acac) 2 催化下的 苯硫酚与 2-甲基丁-3-炔-2-醇间的炔烃氢硫化反应(产率 在 $27 \% \sim 47 \%$ 之间) (Figure 10).

同年, Hübner 团队 ${ }^{[28]}$ 利用 $3 \mathrm{D}$ 打印技术设计了一种 气密 NMR 管/旋转器组合装置和比色杯. 这两种 $3 \mathrm{D}$ 打 印装置都是以聚酰胺为原料, 在充满惰性气体的环境中 打印而成. 他们利用该装置对芳基卤化物和芳基丙酸间 的 Sonogashira 偶联反应进行研究, 且获得了比较高的 产率(60\% 99\%) (Figure 11).
(A) Suzuki-Miyaura cross coupling

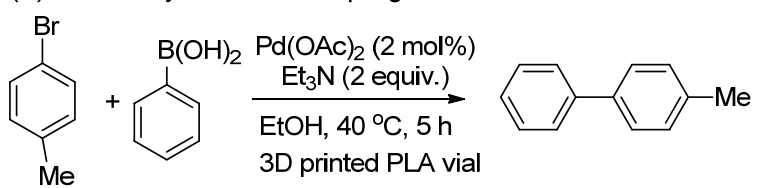
standard glass (79\%), ABS (54\%) PETG (53\%), PLA (50\%), PP (42\%)

(B) Hydrothiolation of alkyness

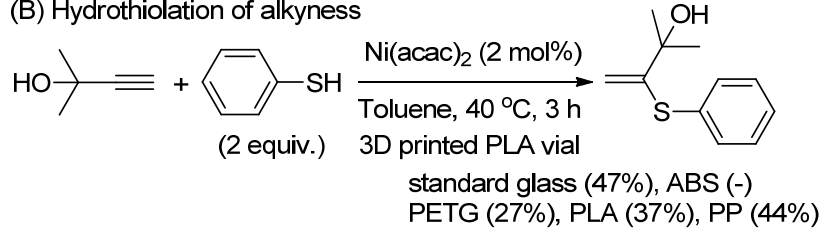

图 10 Suzuki-Miyaura 交叉耦合反应(A)及炔烃硫氢化反应 (B)

Figure 10 Suzuki-Miyaura cross coupling (A) and hydrothiolation of alkyness (B)

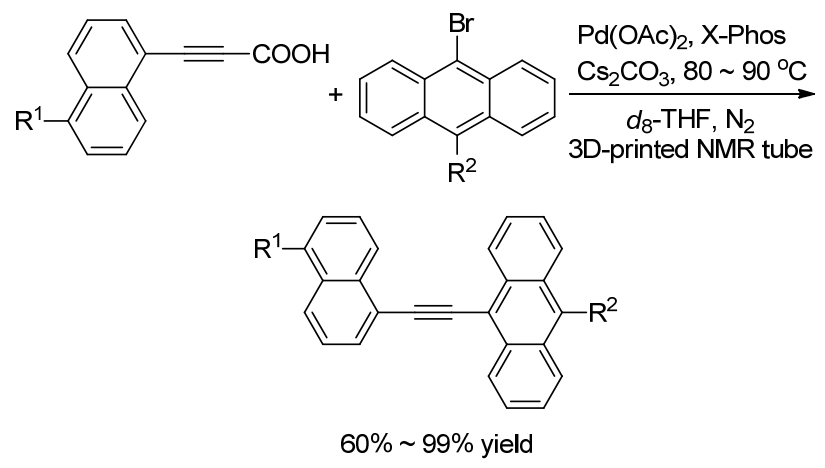

图 11 Sonogashira 偶联反应

Figure 11 Sonogashira coupling reaction

\section{3D 打印流反应器}

2012 年, Cronin 团队 ${ }^{[29]}$ 首次报道了以聚丙烯(PP)为 打印原材料，基于 3D 打印技术的微流反应器制备方法. 此反应器是一种由内径为 $0.8 \mathrm{~mm}$ 的圆通道组成、容积 约为 $270 \mathrm{~mL}$ 的三进口微反应装置. 该团队用这一微流 反应器进行了以下两个反应: (1)茮胺和苯甲醛在甲醇溶 液中反应形成相应的亚胺化合物后，再用氰基嗍氢化钠 将其还原生成二苄亚胺，产率高达 100\%；(2)茮胺、苯甲 醛和氰基硼氢化钠于甲醇溶液反应后，再与烯丙基溴反 应生成相应的仲胺和叔胺混合物.

随后, Cronin 团队 ${ }^{[30]}$ 又报道了一种改进的新型 PP 材质的连续流反应器. 这一新型反应器是将两个相似的 反应器用含有标准 HPLC 连接器的 PTFE 管串联而成: 第一个反应器是由内径为 $1.5 \mathrm{~mm}$ 的圆通道组成的容积 为 $500 \mathrm{~mL}$ 的二进口装置; 另一个反应器是由内径为 $1.5 \mathrm{~mm}$ 的圆通道组成的容积为 $350 \mathrm{~mL}$ 的二进口装置. 此外, 在这一反应器的末端还连接了监测反应进程的 ART-IR 装置(Figure 12). 该团队用此新型反应器同样进 


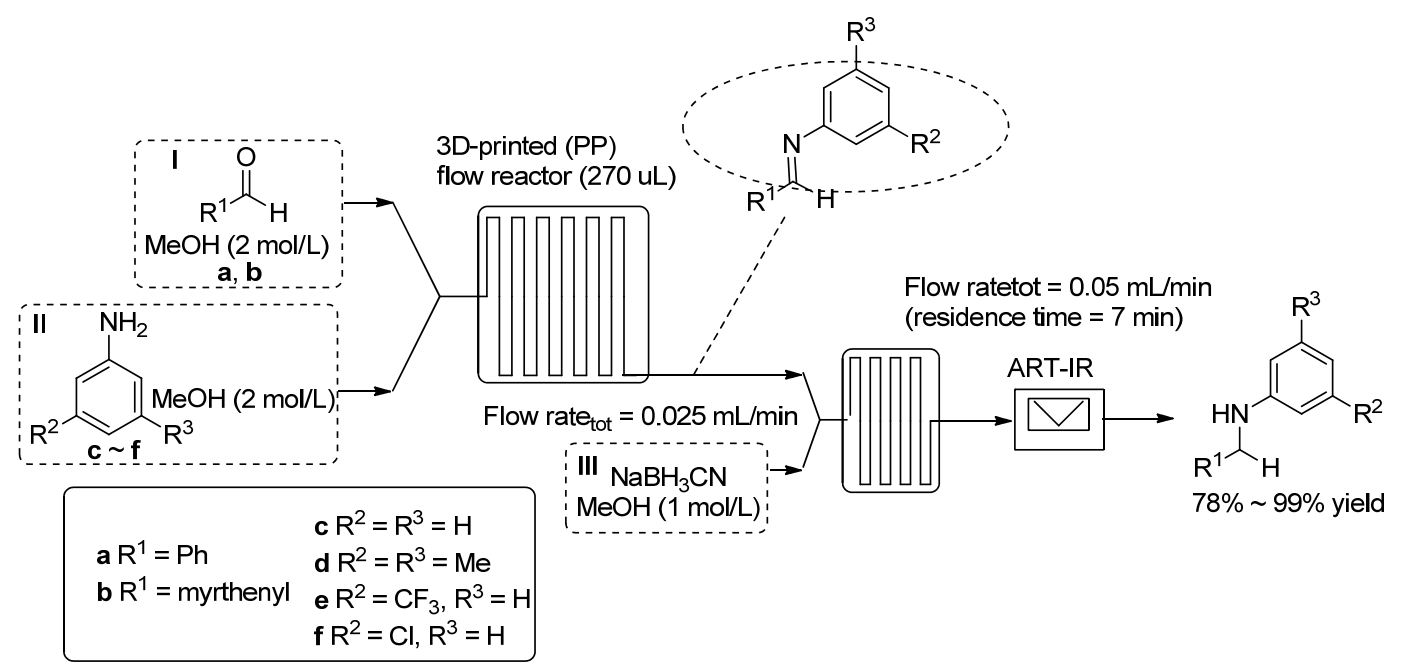

图 12 芳族仲胺的两步合成反应过程

Figure 12 Two-step synthesis of aromatic secondary amines

行了醛、取代苯胺和氰基硼氢化钠还原生成相应亚胺的 反应，结果发现可获得高产率的目标产物(78\% 99\%).

2015 年, Rohr 团队 ${ }^{[31]}$ 基于 SLS 工艺自主设计了一种 长 $200 \mathrm{~mm}$ 、内径 $7 \mathrm{~mm}$ 且具有 $84 \%$ 孔隙率的圆柱形多 孔结构反应器(DPSR). 为了使该反应器获得良好的催 化效果, 他们先在 DPSR 表面上形成 $\mathrm{Al}_{2} \mathrm{O}_{3}-\mathrm{ZnO}$ 涂层, 然后将钯沉积在反应器上. 随后, 该课题组将此微反应 器用于无溶剂条件下 2-甲基-3-丁炔-2-醇(MBY)选择性 氢化制备 2-甲基-3-丁烯-2-醇(MBE)的连续流反应中. 结果表明, 与传统的批反应器相比, 其在选择性、收率 和转化率上都更具有优势.

2017 年, Hornung 团队 ${ }^{[32,33]}$ 报道了一种管式催化静 态反应器(CSM). 这一反应器采用 EBM 工艺, 将一些金 属催化剂(镍、铂)涂覆在钛合金、钴铬合金或不锈钢合 金上制得. 该团队将此反应器置于 Swagelok 不锈钢管 中后, 将其与其它设备(原、氢气瓶等)相连构成连续流 气液加氢反应装置. 他们用该反应装置进行了烯烃、炔 烃、羰基化合物等不饱和化合物的催化氢化还原反应, 结果发现在 $4.5 \sim 6.5 \mathrm{~min}$ 就可达到不低于 $90 \%$ 的转化率. 同年, Christie 团队 ${ }^{[34]}$ 报道了多种主要用于杂环合成且 具有嵌入式分析功能的多功能流体装置. 起初, 该团队 采用 SLA 工艺制造了一种带有联机光谱流动室的反应 器. 这一反应器是由内径为 $1.5 \mathrm{~mm}$ 、容积为 $2.8 \mathrm{~mL}$ 的 圆形通道构成, 并被置于 HPLC 检测器中. Christie 才队 将此反应器与 $5 \mathrm{~mL}$ 不锈钢盘管反应器相连, 并通过 $\mathrm{DAD}$ 检测器的线上分析来确定 $R$-香芹酮和氨基脲在乙 酸钠的甲醇溶液中生成相应缩氨基嫝的最佳反应条件. 研究表明, 温度为 $69{ }^{\circ} \mathrm{C}$ 、流速为 $0.27 \mathrm{~mL} / \mathrm{min}$ 是此反应 的最佳条件. 此后, 该团队以 Ti-6Al-4V 合金粉末为打
印材料, 采用SLM工艺打印了一种新的反应器. 这一反 应器是由内径为 $2 \mathrm{~mm}$ 、容积为 $10 \mathrm{~mL}$ 的圆形通道构成. 将该反应器置于 HPLC 恒温控制室代替不锈钢盘管反应 器进行上述研究, 发现温度为 $79.6{ }^{\circ} \mathrm{C}$ 、流速为 0.24 $\mathrm{mL} / \mathrm{min}$ 是此反应的最佳条件，且可获得产率为 $56 \%$ 的 目标产物.

同年, Benaglia 等 ${ }^{[35]}$ 自制了一个用于立体选择性催 化合成活性药物成分的 3D 打印流反应器. 以 HIPS、 PLA 或尼龙为打印材料, 经 FDM 工艺获得了具有不同 形状和通道尺寸的 3D 打印微流反应器. 该团队首先以 芳醛和硝基乙烷两组分间的 Henry 反应探究了 3D 打印 流反应器的材料、尺寸和几何构型对反应的影响. 在乙 醇为溶剂、温度 $-20{ }^{\circ} \mathrm{C}$ 和停留时间为 $30 \mathrm{~min}$ 的最优反 应条件下, 流反应器的打印材料、尺寸和形状对反应的 最终结果影响不大, 产率、anti/syn 值和 ee 值分别都在 $95 \% 、 70 ： 30$ 和 $82 \%$ 左右. 此外, 该团队将该流反应器 与配备有 $\mathrm{Pd} / \mathrm{C}(10 \mathrm{wt} \%)$ 柱作为催化剂的 ThalesNano H-Cube Mini 装置相连, 组成了一种用于立体选择性催 化合成手性 1,2-氨基醇的多步连续流动装置, 并成功的 合成去甲麻黄碱(产率，90\%; anti/syn, 70：30; $e e_{\text {anti }}$, $81 \%$ ) (Figure 13).<smiles>C[C@@H](N)C(O)c1ccccc1</smiles>

$(1 R, 2 S)$-Norephedrine<smiles>C[C@H](N)C(O)c1cccc(O)c1</smiles>

$(1 R, 2 S)-M e t a r a m i n o l$

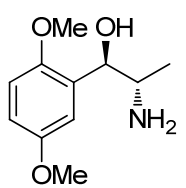

$(1 R, 2 S)$-Methoxamine
图 13 手性 1,2-氨基醇的结构

Figure 13 Structure of chiral 1,2-amino alcohols

2017 年, Hilton 团队 ${ }^{[36]}$ 以 PP 为原料设计了一种具有 
内螺旋管道的 3D 打印柱反应器, 并将其作为一个加热 元件应用于英国 Uniqsisi 公司生产的 FlowSyn 连续流动 反应器中. 该团队首先通过 2-氟硝基苯或 4-氟-3-硝基 苯甲酸甲酯与多种伯胺衍生物间的 $\mathrm{S}_{\mathrm{N}} 2 \mathrm{Ar}$ 反应, 对这种 $3 \mathrm{D}$ 打印柱反应器进行性能探究. 研究表明, 在 $\mathrm{DMF} /$ DIPEA 为溶剂、温度为 $150{ }^{\circ} \mathrm{C}$ 以及流速为 $0.32 \mathrm{~mL} / \mathrm{min}$ 的最优条件下, 此柱反应器对 $\mathrm{S}_{\mathrm{N}} 2 \mathrm{Ar}$ 反应非常适用, 大 多数产物的收率都在 $95 \%$ 以上, 并且其循环使用次数可 达到 5 次. 此外, 他们又将此反应器用于分子内酰基环 化(IAC)反应来合成一些与天然产物(如刺桐生物碱、 Lycorane 生物碱)相关的二环或四环的杂环化合物. 结 果发现, 在 $80{ }^{\circ} \mathrm{C}$ 、流速为 $0.1 \mathrm{~mL} / \mathrm{min}$ 的微波条件下, 在 该柱反应器中进行的 IAC 反应具有非常好的收率, 二环 杂环化合物和四环杂环化合物的收率分别为 $34 \%$ $77 \%$ 、45\% 54\% (Figure 14).

最近, Bettermann 团队 ${ }^{[37]}$ 通过熔融沉积成型工艺 (FDM) 设计了一种 $3 \mathrm{D}$ 打印管状弯曲反应器. 这一反应 器在 $210{ }^{\circ} \mathrm{C}$ 的喷嘴温度下以聚乳酸纤维为打印材料逐 层打印而成, 且由内径为 $18 \mathrm{~mm}$ 、总长为 $1340 \mathrm{~mm}$ 的弯 管构成. 该团队将此反应器与空气冷却系统、计量葲等
相连，构成一个用于连续乳液共聚合的反应装置，并用 该反应装置成功地进行了苯乙烯/丙烯酸丁酯和乙酸乙 烯酯/新癸酸乙烯酯氧化还原引发下的乳液共聚反应, 获得了 $81 \% \sim 99 \%$ 的转化率. 另外，该乳液聚合反应装 置还具有以下优点: (1)停留时间分布短而窄、死体积少 和流动性能非常适宜; (2)不会出现污染、堵塞和变形等 情况; (3)可通过热成像技术观察乳液聚合过程.

2018 年, Genet 团队 ${ }^{[38]}$ 设计了一种 $3 \mathrm{D}$ 打印金属催化 静态混合器(Pd-CSM、Ni-CSM). 先通过冷喷涂沉、电 镀沉积等沉积方法分别将镍和钯负载到该静态混合器 表面, 然后将其塞入内径为 $6 \mathrm{~mm}$ 、长为 $150 \mathrm{~mm}$ 的钢管 而形成. 该团队在 Pd-CSM 和 Ni-CSM 反应器上分别进 行了醛或酮与伯胺两组分间的单步连续流还原胺化反 应，结果显示大多数合成产物反应的转化率都在 $80 \%$ 以 上(Figure 15). 此外, 他们将 Vapourtec R2/R4 管式流动 反应器与 CSM 反应器相连组成一个新的反应系统, 并 在该系统中进行了两步连续流还原胺化反应, 也获得较 高的产率(79\% 85\%) (Figure 16). 可见, 该静态混合器 对还原胺化反应具有高催化活性.

(A) IAC cyclization for bicyclic heterocycles

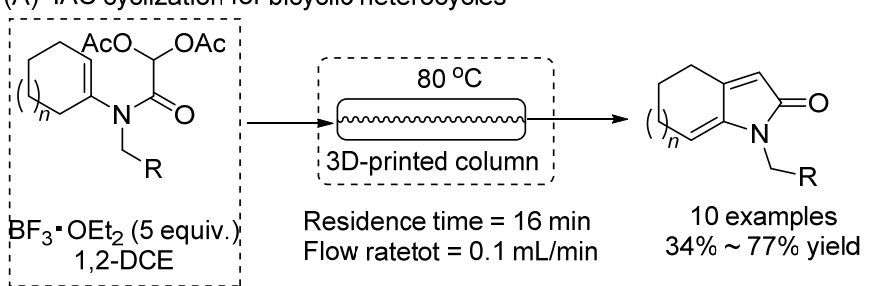

(B) IAC cyclization for the synthesis of tetracyclic heterocycles

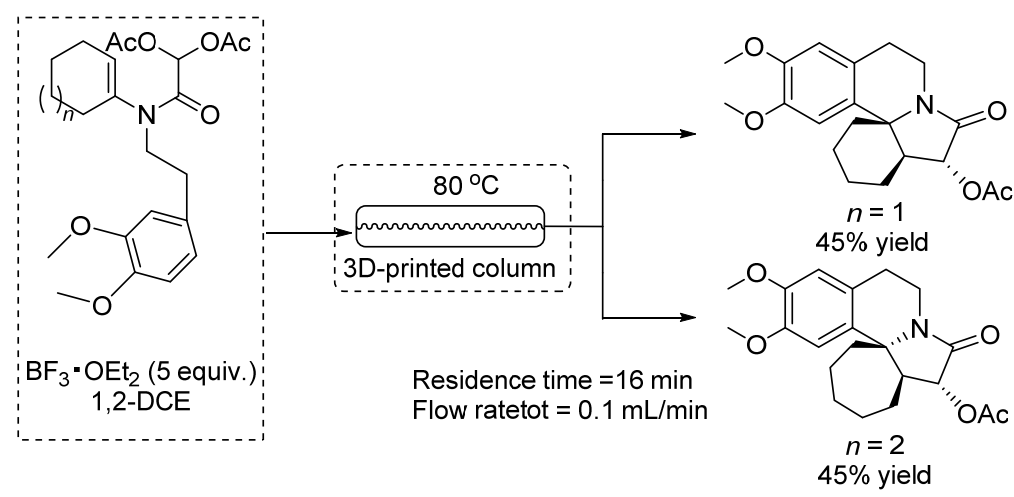

图 14 多杂环化合物合成的分子内环化过程

Figure 14 Intramolecular cyclization of synthetic polycyclic heterocyclic compounds for the synthesis of alkaloids precursors

$$
\stackrel{\mathrm{R}^{2}}{\mathrm{R}^{3}}+\mathrm{R}^{1}-\mathrm{NH}_{2} \frac{\mathrm{H}_{2}}{\text { Ni-CSM reactor or Pd-CSM reactor }} \underset{>80 \% \text { yield }}{\stackrel{\mathrm{R}^{1}}{\mathrm{R}^{2}}} \stackrel{\mathrm{R}^{3}}{\mathrm{R}^{2}}
$$

图 15 醛和酮于 CSM 反应器中的一步还原胺化反应

Figure 15 One-step reductive amination of aldehydes and ketones in a CSM reactor 


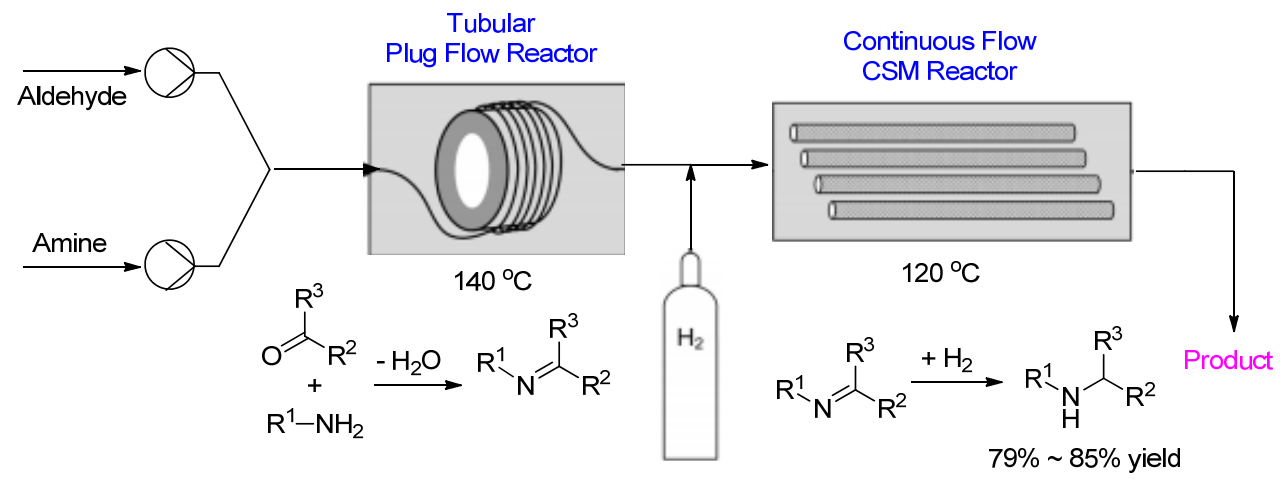

图 16 酫和酮的两步连续流动还原胺化反应流程简图

Figure 16 Reductive amination using a two-step continuous flow procedure

2018 年, Kitson 团队 ${ }^{[39]}$ 报道了一种独立的自动化塑 料串联式反应装置. 这一反应装置以 PP 作为打印材料, 并在 ChemCAD 中设计并创建出来. 该反应装置包括间 歇反应、相分离、溶剂蒸发和过滤等操作过程, 且主要 应用于精细化学品和药品的制造. 该团队应用该反应装 置更加高效的合成了拉莫三嗪(每步产率: $73 \%, 76 \%$ )、 (土)-巴氯芬(每步产率: $59 \%, 95 \%$, quant.)和佐利咪唑(每 步产率: $87 \%, 85 \%, 77 \%$ )三种药物分子, 并与在传统玻 璃反应器中进行的相同药物合成反应进行了比较. 研究 表明, 相对于玻璃反应器, 该反应装置中进行的药物合 成反应具有更高的产率. 此外, 这一研究为无专业设施 下的药品生产提供了可行性.

\section{5 总结与展望}

近年来，随着 3D 打印技术在有机合成领域应用的 不断深入，3D 打印技术不仅可很好地获得具有可控比 表面积的任何构型载体, 还可快速实现具有特殊用途的 特定反应器的打印, 为科研人员进行化学研究提供了更 加灵活丰富的手段. 目前, 基于 3D 打印技术的有机合 成虽然是一个新兴的研究方向, 但在化学工作者的不解 努力下，已经在异相催化剂、微流反应器等有机合成方 向上取得非常好的进展, 成功地证明了 3D 打印技术与 有机合成这两个不同领域的结合是可行且具有非常好 的发展前景. 然而, 不论是 3D 打印异相催化剂或异相 催化剂载体、反应装置还是流反应器, 大多研究只是将 3D 打印技术应用到反应研究过程中的某一部分, 并未 涉及到反应研究的全过程. 此外, 高的打印成本、打印 材料的限制以及现今技术的不成熟等因素使其的应用 难以普及化 ${ }^{[40]}$, 尤其是有限的打印材料使其在有机合 成领域中的扩大应用受到挑战. 因此, 开发新的打印材 料、降低 3D 打印技术的应用成本和扩大 $3 \mathrm{D}$ 打印技术 的应用范围, 是该研究方向在今后发展中重要的课题挑 战. 面对实验室的小批量生产制造, 可采用自组装技术 和使用开源软件在一定程度上来降低 $3 \mathrm{D}$ 打印机的成本.
而在新打印材料的开发研究方面，不同材料混合、材料 的化学改性等是改变材料特性以获得期望材料的重要 手段. 总之, 3D 打印技术作为一种可以制造复杂反应装 置、各种新型高效催化剂等的新方法，其在有机合成领 域中具有巨大的发展空间与发展潜力.

\section{References}

[1] Hull, C. W. US 04575330, 1986.

[2] Huang, W. D. J. New Industrialization 2016, 6, 53 (in Chinese). (黄卫东，新型工业化, 2016, 6, 53.)

[3] Gunther, D.; Heymel, B.; Gunther, J. F.; Ederer, I. Rapid Prototyping J. 2014, 20, 320

[4] Ding, Y. C. Minying Keji 2018, (7), 166 (in Chinese). (丁有成，民营科技, 2018, (7), 166.)

[5] Zhang, M.; Jiang, M.-H.; Wu, G.-H.; Yang, Q.-L. Beverage Ind 2015, 18, 57 (in Chinese). (张明, 姜明洪, 吴光虹, 杨秋玲, 饮料工业, 2015, 18, 57.)

[6] Jing, L. G.; Shen, L. J. Biology Teaching 2016, 41, 6 (in Chinese). (井乐刚, 沈丽君, 生物学教学, 2016, 41, 6.)

[7] Hu, Y. P. Mech. Res. Appl. 2016, 29, 193 (in Chinese). (胡彦萍, 机械研究与应用, 2016, 29, 193.)

[8] Zhu, G.; Mo, W.-J. Val. Eng. 2015, 34, 178 (in Chinese). (朱阁,莫蔚靖, 价值工程, 2015, 34, 178.)

[9] Paulsen, S. J.; Miller, J. S. Dev. Dynam. 2015, 244, 320

[10] He, C. L.; Tang, Z. H.; Tian, H. Y.; Chen, X. S. Acta Polym. Sin. 2013, (6), 722 (in Chinese) (贺超良，汤朝晖，田华雨，陈学思，高分子学报, 2013，(6), 722.)

[11] Zhang, X. J.; Tang, S. Y.; Zhao, H. Y.; Guo, S. Q.; Li, N.; Chen, B. Q. J. Mater. Eng. 2016, 44, 122 (in Chinese). (张学军, 唐思熠, 肇恒跃, 郭绍庆, 李能, 孙兵兵, 陈冰清, 材 料工程, 2016, 44, 122.)

[12] Chen, S. P.; Yi, H.-P.; Luo, Z. H.; Zhu Ge, X. Q.; Luo, K. Mater Rev. 2016, 30, 54 (in Chinese). (陈硕平，易和平，罗志虹，诸葛祥群，罗鲲，材料导报，2016, 30, 54.)

[13] Li, X.-L.; Ma, J.-X.; Li, P.; Chen, Q.; Zhou, W.-M. Proc. Autom. Instrum. 2014, 35, 1 (in Chinese). (李小丽，马剑雄，李萍，陈琪，周伟民，自动化仪表，2014，35, 1.)

[14] Rossi, S.; Puglisi, A.; Benaglia, M. ChemCatChem 2018, 10, 1512.

[15] Tubío, C. R.; Azuaje, J.; Escalante, L.; Coelho, A.; Guitián, F.; Sotelo, E.; Gil, A. J. Catal. 2016, 334,110.

[16] Azuaje, J.; Tubío, C. R.; Escalante, L.; Gómez, M.; Guitián, F.; Coelho, A.; Caamaño, O.; Gil, A.; Sotelo, E. Appl. Catal. A-Gen. 2017, 530, 203.

[17] Manzano, J. S.; Weinstein, Z. B.; Sadow, A. D.; Slowing, I. I. ACS Catal. 2017, 7, 7567.

[18] Díaz-Marta, A. S.; Tubío, C. R.; Carbajales, C.; Fernandez, C.; Es- 
calante L.; Sotelo, E.; Guitian, F.; Barrio, V. L.; Gil, A.; Coelho, A. ACS Catal. 2018, 8, 392.

[19] Symes, M. D.; Kitson, P. J.; Yan, J.; Richmond, C. J.; Cooper, G. J.; Bowman, R. W.; Vilbrandt, T.; Cronin, L. Nat. Chem. 2012, 4, 349.

[20] Johnson, R. D. Nat. Chem. 2012, 4, 338.

[21] Kitson, P. J.; Symes, M. D.; Dragone, V.; Cronin, L. Chem. Sci. 2013, 4, 3099

[22] Kitson, P. J.; Glatzel, S.; Chen, W.; Lin, C. G.; Song, Y. F.; Cronin, L. Nat. Protoc. 2016, $11,920$.

[23] Mathieson, J. S.; Rosnes, M. H.; Sans, V.; Kitson, P. J.; Cronin, L. Beilstein J. Nanotechnol. 2013, 4, 285.

[24] Kitson, P. J.; Glatzel, S.; Cronin, L. Beilstein J. Org. Chem. 2016, 12, 2776.

[25] Zalesskiy, S. S.; Shlapakov, N. S.; Ananikov, V. P. Chem. Sci. 2016, 7,6740 .

[26] Kucherov, F. A.; Gordeev, E. G.; Kashin, A. S.; Ananikov, V. P. Angew. Chem. 2017, 29, 16147.

[27] Gordeev, E. G.; Degtyareva, E. S.; Ananikov, V. P. Russ. Chem. B 2016, 65, 1637.

[28] Lederle, F.; Meyer, F.; Kaldun, C.; Namyslo, J. C.; Hübner, E. G. New J. Chem. 2017, 41, 1925.

[29] Kitson, P. J.; Rosnes, M. H.; Sans, V.; Dragone, V.; Cronin, L. Lab Chip. 2012, 12, 3267.

[30] Dragone, V.; Sans, V.; Rosnes, M. H.; Kitson, P. J.; Cronin, L. Beilstein J. Org. Chem. 2013, 9, 951.
[31] Elias, Y.; von Rohr, P. R.; Bonrath, W.; Medlock, J.; Buss, A. Chem. Eng. Process 2015, 95, 175.

[32] Avril, A.; Hornung, C. H.; Urban, A.; Fraser, D.; Horne, M.; Veder, J. P.; Tsanaktsidis, J.; Rodopoulos, T.; Henry, C.; Gunasegaram, D. R. React. Chem. Eng. 2017, 2, 180.

[33] Hornung, C. H.; Nguyen, X.; Carafa, A.; Gardiner, J.; Urban, A.; Fraser, D.; Horne, M. D.; Gunasegaram, D. R.; Tsanaktsidis, J. Org. Process Res. Dev. 2017, 21, 1311.

[34] Capel, A. J.; Wright, A.; Harding, M. J.; Weaver, G. W.; Li, Y.; Harris, R. A.; Edmondson, S.; Goodridge, R. D.; Christie, S. D. Beilstein J. Org. Chem. 2017, 13, 111.

[35] Rossi, S.; Porta, R.; Brenna, D.; Puglisi, A.; Benaglia, M. Angew. Chem. 2017, 129, 4354.

[36] Rao, Z. X.; Patel, B.; Monaco, A.; Cao, Z. J.; Barniol-Xicota, M.; Pichon, E.; Ladlow, M.; Hilton, S. Eur. J. Org. Chem. 2017, 44, 6499.

[37] Bettermann, S.; Schroeter, B.; Morit, H.-U.; Pauer, W.; Fassbender, W.; Luinstra, G. A. Chem. Eng. J. 2018, 338, 311.

[38] Genet, C.; Nguyen, X.; Bayatsarmadi, B.; Horne, M. D.; Gardiner, J.; Hornung, C. H. J. Flow. Chem. 2018, 8, 81.

[39] Kitson, P. J.; Marie, G.; Francoia, J.-P.; Zalesskiy, S. S.; Sigerson, R. C.; Mathieson, J. S.; Cronin, L. Science 2018, 359, 314.

[40] Huang, J.; Jiang, S. Adv. Mater. Ind. 2013, (1), 62 (in Chinese). (黄健，姜山，新材料产业, 2013, (1), 62.)

(Cheng, F.) 